

\section{DISCLAIMER}

This report was prepared as an account of work sponsored by an agency of the United States Government. Neither the United States Government nor any agency Thereof, nor any of their employees, makes any warranty, express or implied, or assumes any legal liability or responsibility for the accuracy, completeness, or usefulness of any information, apparatus, product, or process disclosed, or represents that its use would not infringe privately owned rights. Reference herein to any specific commercial product, process, or service by trade name, trademark, manufacturer, or otherwise does not necessarily constitute or imply its endorsement, recommendation, or favoring by the United States Government or any agency thereof. The views and opinions of authors expressed herein do not necessarily state or reflect those of the United States Government or any agency thereof. 


\section{DISCLAIMER}

Portions of this document may be illegible in electronic image products. Images are produced from the best available original document. 


\title{
BAND BROADENING STUDIES USING THE PARAMETERS FOR AN EXPONENTIALLY MODIFIED GAUSSIAN
}

\author{
R. E. Pauls' and L. B. Rogers
}

\begin{abstract}
The effects on chromatographic peak shape of dead volume and flow rate have been examined using the standard deviation of the Gaussian component of the peak and the exponential decay constant. For a non-retained solute, addition of dead volume led to an increase in the standard deviation that was independent of flow rate while the decay constant was inversely proportional to the flow. Smaller changes were observed for a retained species.
\end{abstract}

IPresent Address: Amoco Research Center, P. O. Box 400, Naperville, Illinois 60540.

"This report was prepared as an account of work sponsored by the United States Govemment. Neither the United States nor the United States Energy Research and Development Administration, nor any of their employees, nor any of their contractors, subcontractors, or their employees, makes any warranty, express or implied, or assumes any legal liability or responsibility for the accuracy, completeness or usefulness of any information, apparatus, product or process disclosed, or represents that its use would not infringe privately-owned rights.". 
BAND BROADENING STUDIES USING

THE PARAMETERS FOR AN EXPONENTIALLY

MODIFIED GAUSSIAN

R. E: Pauls' and L. B. Rogers

Department of Chemistry

University of Georgia

Athens, Georgia 30602

'Present address: Amoco Research Center

P. O. Box 400

Naperville, Illinois 60540 


$$
\begin{aligned}
& \text { ABSTRACT } \\
& \text { The effects on chromatographic peak shape of dead volume and flow rate have } \\
& \text { been examined using the standard deviation of the Gaussian component of the peak } \\
& \text { and the exponential decay constant. For a non-retained solute, addition of dead } \\
& \text { volume led to an increase in the standard deviation that was independent of flow } \\
& \text { rate while the decay constant was inversely proportional to the flow. Smaller } \\
& \text { changes were observed for a retained species. }
\end{aligned}
$$




\section{INTRODUCTION}

A gais o! romatographic column is normally assumed to act as a Gaussian operator, broadening the $\delta$ input into a Gaussian distribution as it passes through the column. However, pure Gaussian peaks are not found experimentally. This is because non-column factors such as dead volume, detector. time-constants and injection profile convolute the Gaussian distribution. Schmauch (1), as well as Johnson and Stross (2), have shown that detector dead-volume will exponentially modify a chromatographic peak. McWilliam and Bolton (3) have also shown that time constants of detector--amplifier systems will exponentially convolute a Gaussian input profi le. For these reasons, an exponentially modified Gaussian has been widely used as a model for chromatographic peak shapes

Several workers $(4,5,6)$ have applied an exponentially modified Gaussian as a model in the least-square fitting and deconvolution of chromatographic peaks. An exponentially modified Gaussian is generated by the following integral:

$$
f(t)=\frac{N}{\tau \cdot \sigma \sqrt{2 \pi}} \int_{0}^{\infty} \exp \left[-\frac{\left(t-t R^{-t^{\prime}}\right)^{2}}{2 \sigma^{2}}\right] \exp \left[\frac{-t^{\prime}}{\tau}\right] d t
$$

where $N$ is the peak amplitude, $\underline{t}_{-}$is the center of gravity of the Gaussian component, $\sigma$ is the standard deviation of the Gaussian, $T$ is the time constant of the exponential decay and $t^{i}$ is a dummy variable of integration. The width 
of an exponentially modified Gaussian has two components: o, a symmetrical component due to the original Gaussian distribution and, $\tau$, a non-symmetrical contribution due to the exponential decay. These two width terms are additive to give the second moment or peak variance.

$$
\underline{M_{2}}=\sigma^{2}+\tau^{2}
$$

Sternberg (7) has published a comprehensive review of extra-column broadening and discussed the contributions to the peak variance of input profile, connecting tubing, and detector time constants. He distinguished contributions from mixing chambers, diffusion chambers, and tubing-diameter expansions. Several workers $(1,2,8,9)$ have published on extra-column factors, especially on the effects on column efficiency of dead volume, such as connecting tubing, fittings and detector volume. Perhaps the most extensive experimental study on the effects of dead volume on column efficiency has been that by Maynard and Grushka (10). In that work they showed that pre-column dead volume degraded column efficiency more than post-column dead volume. They also showed that dead-volume effects were much larger for non-retained species, and that expansions in tubing diameter seriously affected column performance even if the expansion occurred after the column.

The purpose of this study was to briefly examine the effect of precolumn dead volume on the values of $\sigma$ and $T$ for a chromatographic peak. Postcolumn effects were not examined because they were reported to be small (10). 


\section{EXPERIMENTAL}

Reagents

Helium (Selox, Inc ) was used as the carrier gas after it had been purified by. passage over a molecular sieve. Methane and $n$-pentane (spectrophotometric grade, Aldrich Corp., E. Rutherford, NJ) were used as solutes. Chromosorb W; 100/120 mesh, and SE-30 silicone stationary phase were obtained from Alltech Associates (Arlington Heights, IL)。

\section{Apparatus}

A large chromatographic oven described earlier (11) was used in this study. The temperature in the oven was controlled by a Thermatrol proportional controller (Hallikainen Instrument Co., Richmond, CA). The oven temperature was measured using a platinum resistance thermometer (Omega Engineering Inc., Stamford, CT) in conjunction with a digital multimeter (Keithley Instruments, Cleveland, $\mathrm{OH}$ ).

Carrier gas flow was controlled by a Millaflow pressure regulator (Veriflow Corp., Richmond, CA) and a manual flow controller (Porter Instrument Co., Hatfield, PA). Column head pressure was measured using a Heise pressure gauge.

Samples were injected using a $0.5 \mu$ intemal loop sampling valve (Seismograph Service Co., Tulsa, OK). The output current of the flame ionization detector (1800 series, Varian Aerograph, Walnut Creek, CA) was fed to an electrometer (Varian Aerograph, Walnut Creek, CA), amplified, and sent to a multichannel analog-to-digital convertor (Anscan Model 3700, Beckman Instruments, Fullerton, CA). A PDP 11/20 (Digital Equipment Corp., Maynard, MA) was used to control the chromatograph and to carry out all calculations. 
Procedures

Chromosorb W was sieved to size, acid-washed and silanized according to the procedure of Leibrand and Dunham (12). It was then pan-coated with SE-30 to give a $10 \%$ load before being packed into a $150 \mathrm{~cm} \times 2.1 \mathrm{~mm}$ i.d. stainless steel column. The column temperature was held at $71.5^{\circ} \mathrm{C}$. The volume flow of carrier gas was measured using a soap bubble meter.

There was about $70 \mu l$ of dead volume in the form of $0.75 \mathrm{~mm} \mathrm{i.d.} \mathrm{tubing}$ before the column and $60 \mu$ l of dead volume in the $0.5 \mathrm{~mm}$ tubing that connected the column to the detector. Either a $2.1 \mathrm{~mm} \mathrm{i.d.} \times 10 \mathrm{~cm}$ or $5.3 \mathrm{~mm} \mathrm{i.d.} \times 6.6 \mathrm{~cm}$ piece of tubing was added as extra dead volume before the column. The first contributed $0.40 \mathrm{ml}$ of dead volume while the latter contributed $1.67 \mathrm{ml}$ of volume.

After a sample had been injected, there was an initial delay before data were recorded across the peak, usually at rates from 6 to $30 \mathrm{~Hz}$, depending on the peak width. Five runs were made at each flow.

The values of $\tau, \sigma$ and $\underline{H}$ were calculated at each of 3 flow rates with no added dead volume, with $2.1 \mathrm{~mm}$ diameter tubing, and with $5.3 \mathrm{~mm}$ diameter tubing before the column. The same calculations were made at one flow rate for pentane with no extra dead volume and with $5.3 \mathrm{~mm}$ tubing added before the column. The values of $\tau$ and $\sigma$ were calculated in units of both time and volume.

\section{Calculations}

The zeroth through fourth moments were calculated using the appropriate summations (13). From these, the skew and excess of the peak could be calculated. A threshold technique was used to initiate and terminate the summations (14).

The values of $\tau$ and $\sigma$ were calculated by a technique developed by $W . W$. Yau (15). Once the values of $\tau$ and $\sigma$ were known, it was possible to calculate the 
higher moments and the skew and excess. The values for the second through fourth moments are given by (16)

$$
\begin{aligned}
& \underline{M}_{2}=\sigma^{?}+\tau^{2} \\
& \underline{M}_{3}=2 \tau^{3} \\
& \underline{M}_{4}=3 \sigma^{4}+6 \sigma^{2} \tau^{7}+9 \tau^{4}
\end{aligned}
$$

The height equivalent to a theoretical plate, $\underline{H}$; was calculated by

$$
\underline{H}=\frac{\underline{L} W_{1} / 2^{2}}{5.54 t^{2}}
$$

where $\underline{L}$ is the column length, $\underline{W}_{1} / 2$ is the width at half height and $t$ is the time of the peak maximum, as determined by a least-squares fit of the top of the peak.

The volume flow was corrected for compressibility effects using the compressibility factor, $i$, for column temperature, and for the vapor pressure of water.

\section{RESULTS}

Simulation Studies

The values of $\tau$ and $\sigma$ reported here were reproducible to $1 \%$ or better. Preliminary simulation studies using noise-free chromatograms generated by Equation 1 showed that we could recover the values of $\tau$ and $\sigma$ from those chromatograms with accuracies of \pm 0.005 to 0.07 units. Values with noise are given in Table 1 . Our ability to accurately fit actual chromatographic data having a signal-to-noise ratio greater than 100 is shown in Fig. 1. This is a superposition of an experimental chromatographic peak with a peak generated from Equation 1 using the values of $\tau$, $\sigma$ and $I_{R}$ recovered from the chromatogram. The chromatogram was obtained with a 
section of $5.3 \mathrm{~mm} \mathrm{i.d.} \times 6.6 \mathrm{~cm}$ tubing prior to the column, which accounts for the pronounced tail of the peak. The $\tau / \sigma$ ratio for the experimental peak was 4.04.

Experimental Studies

The results of this study are summarized in Table II. Our results for changes in $\underline{H}$ are in good qualitative agreement with those reported by Maynard and Grushka (10), who studied the effects of dead volume on column efficiency as measured by the $\underline{H}$. For example, using methane which was not retained, the addition of a section of $2.1 \mathrm{~mm} \mathrm{i.d}$. tubing before the $2.1 \mathrm{~mm} \mathrm{i.d.} \mathrm{column} \mathrm{had} \mathrm{little} \mathrm{or} \mathrm{no} \mathrm{effect}$ on the column efficiency. Likewise, the addition of $5.3 \mathrm{~mm} i$.d. tubing before the. column had a very large effect on the efficiency, increasing the $\mathrm{H}$ by a factor of 4 to 9 , depending upon the flow rate. The larger effect occurred at the lower flow rate.

Although we used pentarie instead of heptane, our results for changes in $\underline{H}$ were, again, in qualitative agreement with the earlier study (10). The $\underline{H}$ for pentane with no extra dead volume was about $20 \%$ lower than the value for methane, and it, too, was seriously affected by the addition of the large diameter dead volume. However, it increased only by a factor of 3 , much smaller than the nine-fold increase observed for methane. Maynard and Grushka observed an even smaller increase, about $10 \%$, in the $\mathrm{H}$ when using heptane as a solute at $50^{\circ} \mathrm{C}$.

However, interesting differences in the effects were observed when changes in values for $\sigma$ and $\tau$ were examined. The symmetrical broadening of the peak is reflected by the value of $\sigma$. As expected, the value of $\sigma$ for methane, with no added dead volume, increased as the volume flow decreased. In contrast, the increased retention time at lower flow rates offset the increased broadening so, the $\underline{H}$ remained 
fairly constant. When $2.1 \mathrm{~mm}$ i.d. dead volume was added before the column, the value of $\sigma$ increased slightly. In terms of volume units, the increase at different flow rates was roughly constant, about $0.013 \mathrm{ml}$. This corresponded to about a $15 \%$ increase. In contrast, the increased band broadening did not appear as an increase in the $\underline{H}$ because the retention times also increased because of the added volume in added tubing. Larger increases in $\sigma$ were observed when the $5.3 \mathrm{~mm}$ i.d tubing was added before the column. Again, in volume units, the increase, about $0.170 \mathrm{ml}$, was independent of flow rate and corresponded to an increase of 2-3 fold. The largest percentage increase in o occurred at the higher flows, while the largest percentage increase in $\underline{\mathrm{H}}$ occurred at the lower flows.

As expected, pentane had a larger $\sigma$ value than methane at the same flow with no extra dead volume. However, the increase in $\sigma$ with the addition of the $5.3 \mathrm{~mm}$ dead volume was $0.14 \mathrm{ml}$, smaller than that observed for methane. Future studies, using solutes having different retention times, would be desirable. In any case, it is clear that the use of $\sigma$ rather than $\underline{H}$ should provide new and, hopefully; more . useful information about peaks.

Exponential or non-symmetrical band broadening is characterized by $\tau$. Its time and volume values, for methane with no dead volume, showed opposing trends. When calculated as a time, the value of. $\tau$ decreased with increasing flow to a constant value of about 0.36 seconds, while $\tau$ in volume units increased with increasing flow. This decrease in $\tau$ in time units with increasing flow rate was. observed by Littlewood and co-workers (4), who determined the value of $\tau$ and $\sigma$ by a non-linear least-squares technique. A similar trend occurred when $2.1 \mathrm{~mm}$ i.d. dead volume was placed before the column. In contrast, when $5.3 \mathrm{~mm}$ i.d. was added, both the time and volume values of $\tau$ decreased with increasing flow. 
The value of $\tau$ increased slightly when $2.1 \mathrm{~mm} \mathrm{i.d.} \mathrm{tubing} \mathrm{was} \mathrm{added} \mathrm{al} \mathrm{though}$ the increase in $\tau$ was less than the increase in $\sigma$, but large increases in $\tau$, ranging from factors of 2.1 to 8.6 , occurred when the $5.3 \mathrm{~mm} \mathrm{i.d}$. tubing was added to the system. The largest increase in $\tau$ occurred at the lowest flow rates, in contrast to the results for $\sigma$, where the largest per cent increase occurred at the highest flow. Therefore, at the lower flow rates, where the $\underline{H}$ was most severely affected, the non-symmetrical broadening was the chief cause of the degraded efficiency. At. $0.26 \mathrm{ml}$ per sec, over $90 \%$ of broadening, as measured by the second moment, resulted from $\tau$ contributions. At $0.41 \mathrm{ml} /$ per sec., this was reduced to $64 \%$. A plot of $\tau$ in volume units vs volume flow rate gave a straight line with a leastsquares slope of $-3: 9 \pm 0.2$ and an intercept of $1.91 \pm 0.09$.

At $0.26 \mathrm{ml} / \mathrm{sec}$, the value of $\tau$ for pentane with no dead volume in the system was about $50 \%$ larger than for methane. Then, when $5.3 \mathrm{~mm} \mathrm{i.d.} \mathrm{dead}$ volume was infroduced, $\tau$ increased 3.7 times whereas, at the same flow rate; $\tau$ increased 8.6 times for methane. Again, this showed the smaller effect of dead volume on retained species.

In summary, the dead volume affected the values of $\tau$ and $\sigma$ differently indicating that different processes were responsible for the different types of band broadening. The increase in $\sigma$ was independent of flow rate while $\tau$ was inversely proportional to the flow rate when the $5.3 \mathrm{~mm} \mathrm{i.d.} \mathrm{dead} \mathrm{volume} \mathrm{was} \mathrm{added.}$

Once the values of $\tau$ and $\sigma$ had been determined, the skew and excess could be calculated from the higher moments by use of Equations 3-5. These are valuable parameters in the characterization of peak shape, but are very sensitive to noise, baseline drift; and truncation errors (17). In Table III, we have compared the precisions for calculating the skew and excess from the normal summation technique to those obtained from the values of $\tau$ and $\sigma$. Generally, using $\tau$ and $\sigma$, 
the precision for skew was better by a factor of 1.5 to 3 , while the precision for the excess was better by a factor of about 7. Note that for he values for lin skew and excess calculated using $T$ and $\sigma$ were larger than those calculated from the nomal summation by a factor of about 10 to $30 \%$ :

\section{DISCUSSION}

The detailed analysis of the peak shape in terms of $\sigma$ and $\tau$ can, compared to $\underline{H}$, yield a large amount of new information on the effects of dead volume on chromatographic peaks. For instance, with the addition of $2.1 \mathrm{~mm} i \cdot d$. dead volume, the $\underline{H}$ hardly changed while the peak $\sigma$ value increased by $15 \%, a$ direction consistent with expectation.

The dead volume affected the symmetrical and non-symmetrical band broadening in different ways. The addition of dead volume prior to the column added a constant volume to the $\sigma$ values. In contrast, over the flow rates used, the increase in the value of $\tau$ was inversely proportional to the volume flow rate when the $5.3 \mathrm{~mm} \mathrm{i.d}$. dead volume was added. This is an indication that different phenomena were responsible for the increases in $\sigma$ and $\tau$.

The increase in $\tau$ with decreasing flow rate was qualitatively in the correct direction but quantitatively very different from what one would expect if the dead volume were acting as a mixing chamber. Stemberg (7) has given an expression for a mixing chamber

$$
\tau=\underline{V} / \mathbf{F}
$$

where $\underline{V}$ is the dead volume and $F$ is the volume flow rate. The effects observed were experimentally much smaller than those expected from Equation 7. Using a dead volume of $1.67 \mathrm{ml}, \tau$ values calculated from Equation 7 were $6-10$ times 
larger than those experimentally observed. For example, for a flow rate of 0.41 $\mathrm{ml}$ per sec the experimental value was $0.772 \mathrm{sec}$ whereas the calculated value was $6.42 \mathrm{sec}$.

\section{ACKNOWLEDGMENT}

We are grateful to Dr.W.W. Yau for the development of the mathematical procedures used in this study and to Henry Stoklosa for help with the computer programming. This work was supported by the U.S. Energy Research and Development Administration through Contract E(38-1)-854 . 


\section{LITERATURE CITED}

1. L. J. Schmauch, Anal. Chem.; 31, 225 (1959).

2. H. W. Johnson and F. H. Stross, Anal. Chem., 31, 357 (1959).

3. I. G. McWilliam and H. C. Bolton, Anal. Chem., 41; 1755 (1969).

4. A. H. Anderson, T. C. Gibb and A. B. Littlewood, J. Chromatogr . Sci., $8,640(1970)$.

5. H. M. Gladney, B. F. Dowden and J. D. Swalen, Anal. Chem., 41, 883 . (1969).

6. S. N. Chesler and S. P. Cram, Anal. Chem., 45, 1354 (1973).

7. J.C. Steinberg, "Advances in Chromatography," Vol. 2, J. C. Giddings and R. A. Keller, Ed.; Marcel Dekker, New York, N.Y., 1966.

8. R. Kieselbach, Anal. Chem., 35, 1342 (1963).

9. S. P. Cram and T. H. Glenn, J. Chromatogr., 112, 329 (1975).

10. V. Maynard and E. Grushka, Anal. Chem., 44, 1427 (1972).

11. R. B. Westerburg, F. J. Van Lenten and L. B. Rogers, Separ. Sci., 10, $593(1975)$.

12. R. J. Leibrand and L. L. Dunham, Res. Dev., 24(9), 32 (1973)。

13. E. Grushka, M. N. Myers, P. D. Schettler and J. C. Giddings; Anal. Chem., 41, 889 (1969).

14. S. N. Chesler and S. P. Cram, Anal . Chem.., 43, 1922 (1971).

15. W.W. Yau, personal communication, Jan., 1976.

16. E. Grushka, Anal Chem., 44, 1733 (1972).

17. P. R. Rony and J. E. Funk, J. Chromatogr. Sci., 9, 215 (1971). 


\section{TABLE I}

RECOVERIES OF $\sigma$ AND T FROM SIMULATED CHROMATOGRAMS

A. No Noise

$\begin{array}{llll}\sigma_{\text {input }} & \tau_{\text {input }} & \ddots \sigma_{\text {calc }} & \tau_{\text {calc }} \\ 20.000 & 0.00 & 20.008 & 0.07 \\ 20.000 & 2.00 & 20.003 & 2.03 \\ 20.000 & 6.00 & 20.002 & 6.01 \\ 20.000 & 10.00 & 20.005 & 9.995\end{array}$

B. $1 \%$ Noise $^{a}$

$\begin{array}{cccc}\sigma_{\text {input }} & T_{\text {input }} & \sigma_{\text {calc }} & { }^{T} \text { calc } \\ 20.000 & 0.00 & 19.76 \pm .12 & -1.4 \pm 3.0 \\ 20.000 & 2.00 & 19.82 \pm .11 & -2.3 \pm 2.1 \\ 20.000 & 6.00 & 19.96 \pm .12 & 5.85 \pm .47 \\ 20.000 & 10.00 & 20.08 \pm .11 & 9.56 \pm .21\end{array}$

a. Peak-to-peak noise relative to peak height. 
Table II

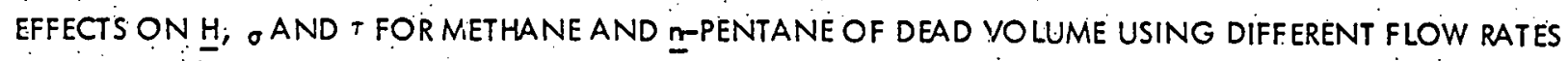

\begin{tabular}{|c|c|c|c|c|c|c|c|c|c|c|c|}
\hline \multirow[b]{2}{*}{ Solute } & & & \multicolumn{3}{|c|}{ No Added Dead Volume } & \multicolumn{3}{|c|}{$2.1 \mathrm{~mm}$ i.d. Dead Volume } & \multicolumn{3}{|c|}{$5.3 \mathrm{~mm} \mathrm{i} \mathrm{d.} \mathrm{Dead} \mathrm{Volume}$} \\
\hline & $\begin{array}{l}\text { Volume Flow } \\
(\mathrm{ml} / \mathrm{sec})\end{array}$ & & $\underline{H}_{(\mathrm{mm})}$ & $\sigma$ & $T$ & $\underline{H}_{(\mathrm{mm})}$ & $\sigma$ & $\tau$ & $\underline{H}(\mathrm{~mm})$ & $\because \sigma$ & $T$. \\
\hline & & $\mathrm{ml}$ & $\therefore$ & 0.072 & 0.148 & & 0.085 & 0.152 & & 0.233 & 0.313 \\
\hline $\mathrm{CH}_{4}$ & 0.41 & sec & 1.034 & $0.179 \pm .003$ & $0.366 \pm .003$ & 1.015 & $0.211 \pm .001$ & $0.377 \pm .001$ & 4.47 & $0.575 \pm .005$ & $0.772 \pm .017$ \\
\hline & & $\mathrm{ml}$ & & 0.086 & 0.135 & & 0.098 & 0.145 & & 0.256 & 0.504 \\
\hline $\mathrm{CH}_{4}$ & 0.37 & sec & 1.026 & $0.233 \pm .003$ & $0.364 \pm .003$ & 1.027 & $0.267 \pm .002$ & $0.391 \pm .001$ & 5.43 & $0.692 \pm .005$ & $1.46 \pm .01$ \\
\hline . & & $\mathrm{m} !$ & & 0.114 & 0.104 & & 0.129 & 0.115 & & 0.286 & 0.902 \\
\hline $\mathrm{CH}_{4}$ & 0.26 & $\sec$ & 1.178 & $0.439 \pm .003$ & $0.400 \pm .004$ & 1.215 & $0.496 \pm .002$ & $0.442 \pm .003$ & 9.65 & $1.10 \pm .01$ & $3.47 \pm .02$ \\
\hline & & $\mathrm{ml}^{-}$ & & 0.173 & 0.159 & & & & & 0.303 & 0.586 \\
\hline $\mathrm{C}_{5} \mathrm{H}_{12}$ & 0.26 & sec & 1.020 & $0.665 \pm .02$ & $0.610 \pm .02$ & & determined & . & 3.29 & $1.167 \pm .003$ & $2.255 \pm .007$ \\
\hline
\end{tabular}


TABLE III

COMPARISON DF THE PRECISION IN THE CALCULATION OF THE SKEW AND EXCESS CALCULATED FROM THE USUAL ("NORMAL") SUMMATIONS AND FROM $T$ AND ${ }_{\sigma}^{\circ}$

$\begin{array}{ccccc}\text { Volume Flow }_{(\mathrm{ml} / \mathrm{sec})} & \text { Skew }_{\text {normal }} & \text { Skcw }_{\tau} & \text { Exccss normal } & \text {. Excess } T \\ 0.26 & 0.48 \pm .02 & 0.590 \pm .006 & 3.56 \pm .08 & 4.18 \pm .01 \\ 0.37 & 1.11 \pm .01 & 1.130 \pm .007 & 4.93 \pm .07 & 6.48 \pm .01 \\ 0.41 & 0.97 \pm 0.01 & 1.331 \pm .003 & 4.63 \pm 0.06 & 5.80 \pm 0.01\end{array}$

a. Solute, methane; $2.1 \mathrm{~mm} \mathrm{i.d.} \mathrm{dead} \mathrm{volume} \mathrm{before} \mathrm{the} \mathrm{column.}$ 


\section{FIGURE CAPTIONS}

Figure 1

Experimental Chromatogram (solid line) and Calculated Points (squares). Column, $2.1 \mathrm{~mm} . \mathrm{i} . \mathrm{d}^{3} \times 150 \mathrm{~cm}-; 10 \%$ SE-30 on $100 / 120$ mesh Chromosorb W; carrier flow, $0.20 \mathrm{ml} / \mathrm{sec}$.; $5.3 \mathrm{~mm}$. i.d. $\times 6.7 \mathrm{~cm}$. tubing before column; sample methane. 


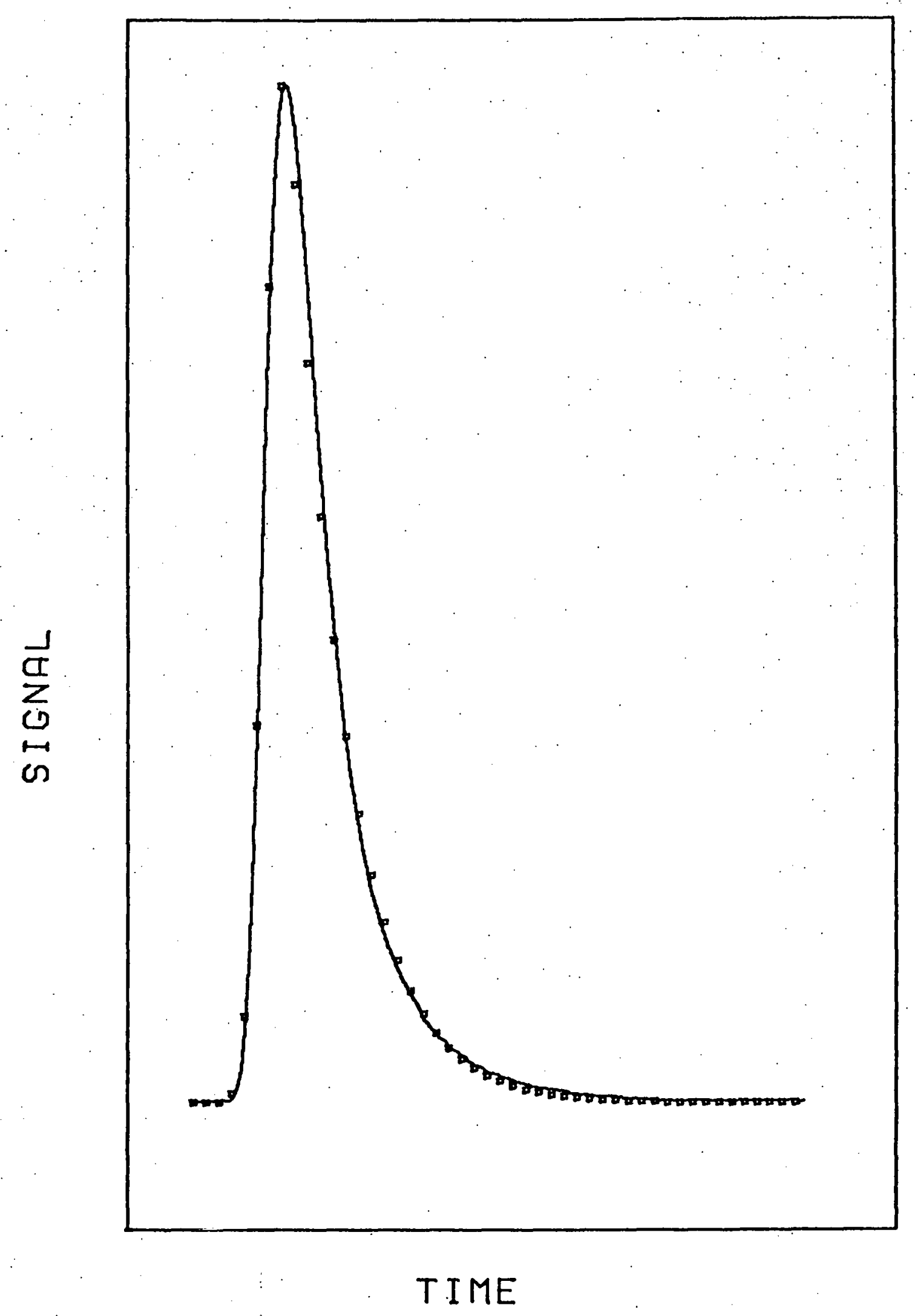

